\title{
The Pathophysiology and Outcomes of Diabetic Patients with Coronavirus Disease 2019 (COVID-19)
}

\author{
Victor Perdana Kusuma1 ${ }^{10}$, Deasy Ardiany ${ }^{2 *}$ iD \\ ${ }^{1}$ Department of Internal Medicine, Faculty of Medicine, Airlangga University, Dr Soetomo General Academic Hospital \\ Surabaya \\ ${ }^{2}$ Endocrinology and Metabolism Division, Department of Internal Medicine, Faculty of Medicine, Airlangga University, Dr \\ Soetomo General Academic Hospital Surabaya
}

\section{A R T I C L E I N F O}

\section{Article history:}

Received 10 August 2021

Received in revised form 17 September 2021

Accepted 19 October2021

Available online 31

October 2021

\section{Keywords:}

COVID-19,

Diabetes Mellitus,

Pathophysiology.

*) Corresponding author: ardianydeasy@gmail.com

\section{A B S T R A C T}

Introduction: Coronavirus Disease 2019 (COVID-19) is a respiratory tract infection caused by the SARS-CoV-2 virus, which was announced a pandemic by the World Health Organization (WHO) on March 11, 2020. On March 2, 2020, two confirmed cases of COVID-19 were initially reported in Indonesia. COVID-19 has been reported in 96.2 million people around the world. COVID-19 has already stolen the lives of almost 2 million individuals. Diabetes mellitus patients face an additional challenge with this disease (DM). Several studies have found a link between diabetes mellitus and COVID-19, as well as a bad prognosis for persons with DM and COVID-19. Aim of this study was to learn more about the link between diabetes and COVID-19, as well as the pathophysiology of diabetes.

Methods: We searched for articles in PubMed and Google Scholar databases till February 2021, with the following keywords: "SARS-CoV-2", "COVID-19", "infection", "pathogenesis", "diabetes"

Results: Diabetes Mellitus increased the severity and mortality of COVID-19 patients due to mechanisms involving inflammation and immune system activation, increased coagulation activity, oxidative stress, glucotoxicity, endoplasmic reticulum stress, renin-angiotensinaldosterone system disorders, apoptotic mechanisms, mitochondrial dysfunction, and damage to pancreatic beta cells. COVID-19 infection can also lead to hyperglycemia and new-onset diabetes.

Conlusion: Diabetes Mellitus is one of the comorbidities linked to a worse COVID-19 prognosis, according to the findings of this literature study. Furthermore, current research suggests that COVID-19 might cause hyperglycemia or new-onset diabetes, which has a poorer prognosis than COVID-19 patients with pre-existing diabetes.

\section{Introduction}

On December 31, 2019, the first case of COVID-19 was reported in Wuhan, Hubei Province, China. The World Health Organization (WHO) then announced COVID-19 a pandemic on March 11, 2020, after announcing a Public Health Emergency of International Concern (PHEIC) on January 30, 2020. On March 2, 2020, two confirmed cases of COVID-19 were initially reported in Indonesia. COVID-19 has been reported in 96.2 million people around the world. COVID-19 has already stolen the lives of almost 2 million individuals. Case incidence remains one of the key drivers of mortality, as seen by a spike in the number of hospitalizations linked to COVID-19 after a large number of cases were reported in a short period of time.,

Diabetes Mellitus is one of the leading causes of illness and mortality in the world, and its prevalence is anticipated to rise dramatically in the future decades. In terms of clinical course and outcome, both diseases have an impact on each other. The goal of this literature review is to better understand the relationship between diabetes and COVID-19, as well as the pathophysiology of diabetes.

\begin{abstract}
Methods
We searched for publications with the following keywords in PubMed and Google Scholar databases till February 2021: "SARS-CoV-2," "COVID-19," "infection," "pathogenesis," and "diabetes." We looked through the WHO's current scientific research and recommendations.
\end{abstract}




\section{Discussion}

COVID-19

Coronavirus disease 2019 (COVID-19) is a respiratory disease caused by the coronavirus family's, severe acute respiratory syndrome coronavirus 2 (SARS-CoV-2) and the genus betacoronavirus. ${ }^{1}$ Coronavirus has single stranded, positive sense RNA genomes that are enormous (up to $30 \mathrm{~kb}$ ). SARS-CoV-2 is more lethal than other endemic viruses, and it is also more lethal to humans than the preceding SARS-CoV-1 and Middle East respiratory syndrome coronavirus (MERS-CoV) explosions in 2003 and $2012 .^{2}$

\section{Pathogenesis}

At first, SARS-CoV-2 outbreak is believed as a result of bat-borne zoonotic transmission. ${ }^{1}$ Human-to-human SARS-CoV-2 transmission can occur directly, primarily through respiratory droplets, when a patient coughs, sneezes, or even talks or sings, or indirectly, when a person touches a SARS-CoV-2-infected surface and then comes into direct contact with mucous membranes such as the eyes, nose, or mouth.5 When SARS-CoV-2 droplets enter the mucosa of the eyes, nose, or mouth. The Spike protein (S) on the virus's surface binds to the ACE2 receptor on respiratory tract cells, inducing fusion of SARS-CoV-2 and the cell membrane via Transmembrane Protease Serine 2. (TMPRSS2). Viruses infecting the cell then release the RNA genome into the cytoplasm, where it is translated into the polyproteins ppla and $1 \mathrm{ab}$, as well as structural proteins. After replication, proteins and viral RNA genomes are formed into virions in the Golgi apparatus's endoplasmic reticulum. These virions are transferred to the cell membrane by vesicles and excreted via exocytosis. ${ }^{6}$

SARS-CoV-2 will migrate to the lower airway and infect bronchial epithelial cells, pneumocytes type I and II, and capillary endothelial cells in the early stages. Increased viral replication will compromise the epithelial-endothelial barrier's integrity. ${ }^{7}$ When $\mathrm{T}$ lymphocytes, monocytes, and neutrophils are recruited, pro-inflammatory cytokines such as interleukin/IL-1, IL-6, IL-10, tumor necrosis factor (TNF-), granulocyte colony stimulating factor, monocyte chemoattractant protein I, and macrophage inflammatory protein I are released. Increased vascular permeability, alveolar wall thickening, pulmonary edema, coagulation activation, and increased $\mathrm{T}$ lymphocyte apoptosis result from the ongoing inflammatory process. A hyaline membrane can form in response to pulmonary edema, resulting in acute respiratory distress syndrome (ARDS). Coagulation activation results in the formation of a microthrombus. Deep Venous Thrombosis (DVT), pulmonary embolism, limb ischemia, stroke infarction, and myocardial infarction are all possible consequences. Because of the presence of ACE2 receptors on the cell surface, SARS$\mathrm{CoV}-2$ is also known to infect the gastrointestinal system, particularly stomach epithelial cells, the duodenum, and the rectum. ${ }^{2,7}$

\section{Clinical Manifestation}

COVID-19 "has a wide clinical spectrum that spans from asymptomatic to severe disease". 8

A. Asymptomatic/pre-symptomatic

The polymerase chain reaction / PCR “testing revealed a positive SARS-CoV-2 result in this group without any clinical symptoms present."8 "Even if the patients do not exhibit clinical symptoms, they might still spread the virus as asymptomatic carriers throughout the incubation phase". 9,10

\section{B. Minor Illness}

Mild symptoms commonly include fever, cough, tiredness, anorexia, and myalgia. Sore throat, nasal congestion, hemoptysis, headache, diarrhea, nausea and vomiting, loss of smell (anosmia), and loss of taste are all possible non-specific symptoms (ageusia) without shortness of breath (dyspnea). 5,7,11 However, some individuals who initially have modest symptoms can experience abrupt clinical deterioration around one week following the symptoms. $^{8}$

\section{C.Moderate Illness}

Patients with moderate symptoms exhibit pneumonia symptoms such as fever, cough, tachypnea, and can be present with or without shortness of breath (dyspnea). The presence of clinical or radiographic signs of lower respiratory tract disease (such as ground glass opacities, consolidation, bronchial wall thickening, or bronchiectasis) with a blood oxygen saturation of 94 percent or above is indicative of moderate sickness. ${ }^{5,7,8,11}$

\section{Severe Illness}

Patients with severe symptoms exhibit signs and symptoms, including febrile, cough, tachypnea, and shortness of breath (dyspnea), as well as one of the following indicators: significant respiratory distress; blood oxygen saturation of less than $94 \%$; respiratory rate of 30 breaths per minute or more; and lung infiltrates of greater than $50 \%$. $^{5,7,8}$

E. Critical Illness

There has been respiratory failure, shock, and multiorgan dysfunction or failure in this group. ${ }^{8}$

\section{COVID-19 Prognosis}

Old age ( $>60$ years), chronic obstructive pulmonary disease (COPD), cardiovascular illness (heart failure, coronary artery disease, or cardiomyopathy), diabetes Mellitus (DM), obesity (BMI 30), sickle cell anemia, chronic renal disease, immunocompromised condition, and cancer are all risk factors for COVID $19 .{ }^{8}$ Those who have comorbidities have a poorer prognosis than patients who do not have comorbidities. Cardiovascular disease, diabetes mellitus, COPD, hypertension, liver disease, renal disease, dementia, and cancer are the most common comorbidities associated with COVID-19 mortality. ${ }^{12}$

\section{Diabetes Mellitus (DM)}

Diabetes Mellitus is "commonly seen as a chronic inflammatory illness caused by a long-period immune system disproportion, metabolic syndrome, or dietary overload linked with obesity." Diabetes Mellitus is one of the main reasons of morbidity globally with 702 death certificates in 2017, and its prevalence is expected to raise dramatically in the future decades. In 2019 , the worldwide prevalence of diabetes is expected to be $9.3 \%$, with a rising trend. Several studies have found that persons with diabetes are more susceptible to infectious illnesses such as Staphylococcus aureus and Mycobacterium tuberculosis. According to research, a 
high plasma glucose levels and diabetes are independent indicators of death and morbidity in SARS patients. A retrospective research in Wuhan, China, discovered that $32 \%$ of 41 COVID-19 patients had comorbidities, and $20 \%$ were diabetic patients. As a result, diabetic people may be more likely to acquire COVID-19 with poorer prognosis. ${ }^{13,14}$

\section{The relationship between COVID-19 and Diabetes Mellitus}

Although the relationship between COVID-19 and DM has yet to be proved, a number of research and theories have been developed in order to identify the probable molecular processes implicated in diabetic COVID-19 patients. As previously stated, diabetes rises the complications of COVID-19 as well as the chance of mortality from COVID-19". Recent data also suggests that diabetic COVID-19 patients are more prone than nondiabetic COVID-19 patients to have more severe COVID 19 symptoms and complications. So far, the mechanism causing worse prognosis in diabetic COVID-19 patients has not been adequately established. According to one theory, hyperglycaemia can enhance viral entrance into cells because Angiotensin-Converting Enzyme-2 (ACE2) and viruses both require glucose to perform their activities. ${ }^{15}$

Furthermore, diabetes patients are commonly treated with Angiotensin-Converting Enzyme Inhibitors (ACEIs) and/or Angiotensin Receptor Blockers (ARBs), both of which can promote increased ACE2 expression in tissues, increasing viral adherence and the vulnerability of severe infection in diabetic patients. ${ }^{16}$

\section{Inflammation and Activation of the Immune System} Inflammation is a physiologic response of the innate immune system to many potentially damaging stimuli. Diabetes is connected with persistent low-grade inflammation and elevated cytokine levels. Toxins,

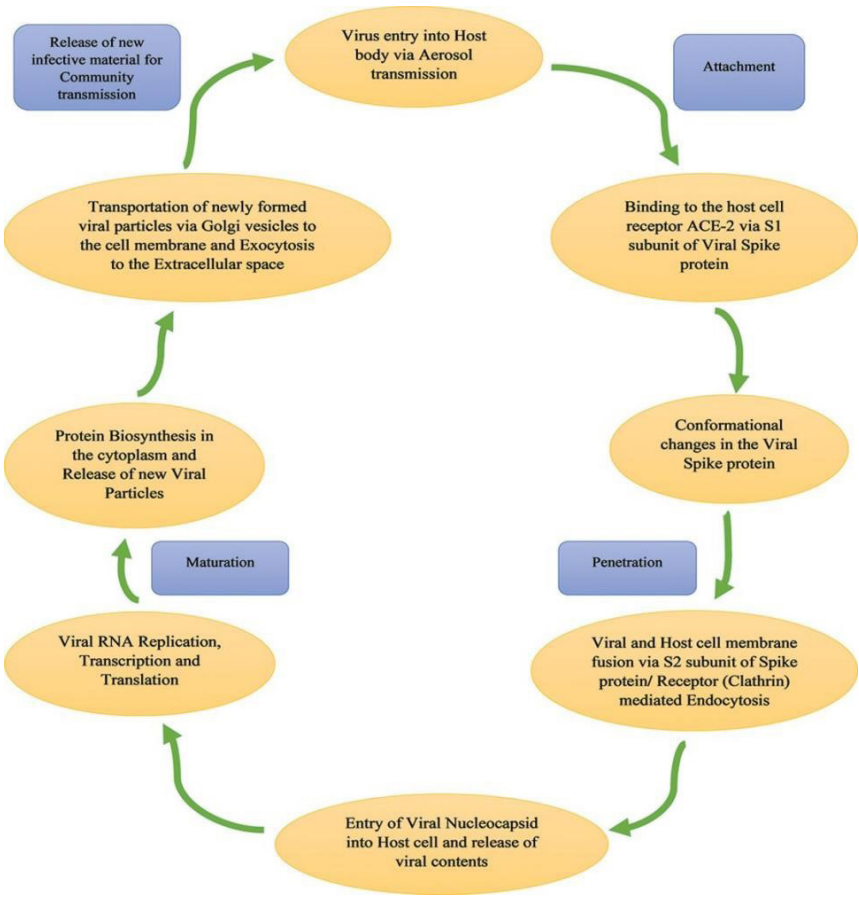

Figure 1. Pathogenesis of COVID-197 irritants, damaged cells, and infections are generally viewed as detrimental stimuli that must be removed in order to preserve homeostasis. TNF-, interleukin (IL), matrix metalloproteinase (MMPs), monocyte chemoattractant protein-1 (MCP-1), transforming growth factor-beta (TGF- $\beta$ ), adhesion molecules, tolllike receptors (TLRs), adipocytokines, and c-reactive protein are all involved in inflammatory responses. ${ }^{17}$ "Acute inflammation is the first phase of the immune unit activity in order to eliminate different hazardous materials from the body. However, chronic inflammation occurs in conditions of impairement immune response in long-period diseases such as diabetes.. ${ }^{18}$ These cytokines have an crucial role in the pathogenesis of many DM complications, increasing the risk of tissue damage. ${ }^{19}$

COVID-19 is a viral infection that causes inflammatory reactions and increased cytokine levels 20. Guo et al discovered that COVID-19 patients with diabetes had considerably greater proportions of several inflammatory cytokines" (including IL-6, CRP, ferritin serum, Coagulation activity, and D-dimer) than COVID-19 patients without diabetes. Chronic inflammation in diabetes can act as a potentiator of the inflammatory response, increasing the chance of cytokine storms in COVID-19 patients. This discovery expresses that individuals with diabetes are "more exposed" to cytokine storms, which can result in "more severe" clinical symptoms.14 A cytokine storm can provoke acute respiratory distress syndrome (ARDS), multi-organ malfunction, physiological function degradation, and even death (Figure 2). ${ }^{14,21}$

\section{Hypercoagulation}

Hypercoagulable condition in COVID-19 patient will result in a poorer prognosis. According to one study, COVID-19 patients with Diabetic had a "higher chance" of hypercoagulability, and many of those with severe or critical symptoms die from pulmonary embolism. ${ }^{16}$

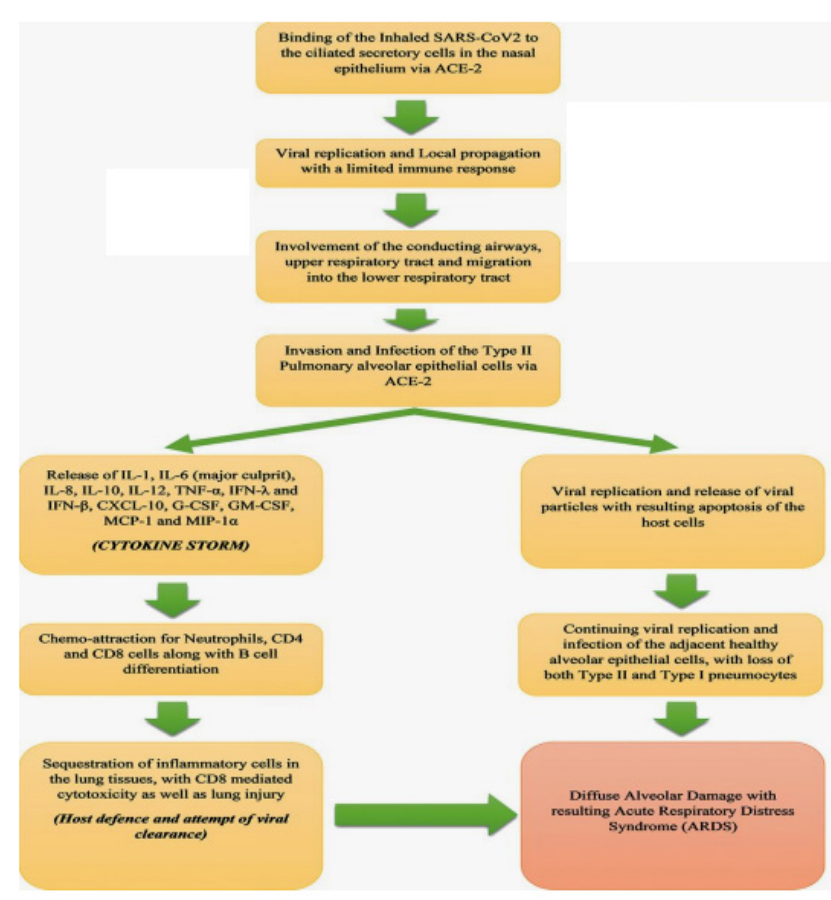

Figure 2. Cytokine storm in COVID-1921 


\section{Oxidative stress}

The"disproportion between free radical species and the efficacy of antioxidant defense mechanisms supporting free radicals"is referred as oxidative stress. It is crucial in the pathophysiology of many DM complications as well as viral respiratory diseases. "This pathologic state begins additional molecular pathways implicated in breathing infections, such as out of control cell damage activity and harming numerous biological components in the respiratory system. Antioxidant supplements is believed to be useful as "supporting therapy" in the treatment of some respiratory infections. ${ }^{15}$

Excessive free radical production is frequently related with Diabetes Mellitus. Uncontrolled diabetes causes oxidative pressure via at the minimum ten molecular mechanisms, including mitochondrial malfunction, cellular antioxidant element depletion, "glucose autoxidation, glycation pathways", lipid peroxidation, activation of free radical promoter enzymes,"sorbitol pathway, protein kinase $\mathrm{C}$ isoforms, hexosamine route, and redox alterations. ${ }^{19,22}$

This oxidative environment may possibly elevate the pathogenicity of viruses such as" coronaviruses. Because SARS-CoV-2 uses "host cell machinery for growing,"the situation of the host cells is a critical aspect of invading pathogen infectivity," and oxidative environment is one of the key variables that might enhance coronavirus reproduction in the host cells. Besides that, Oxidative pressure stimulates additional pathophysiologic processes such as inflammation and cell injury, as well as molecular mechanisms such as mitogen-activated protein kinase (MAPK), which accelerates viral infection in the tissue. Furthermore, oxidative stress may promote coronavirus attack cells by altering their entranceway. Free radicals, for example, have a substantial influence on and change the distribution of the transmembrane protease serine 2 , a key enzyme implicated in nonendosomal viral entry. As a result, oxidative pressure in host cells is a critical point of coronavirus entryway, duplication, and pathogenesis. ${ }^{23}$

\section{Glucotoxicity}

In hyperglycemic conditions, this pathologic mechanism shifts glucose stability through to routes such as polyol, hexosamine, or sorbitol. The harmful effect of an excess of glucose on different metabolic pathways, which is frequent in untreated diabetes, is referred to as glucotoxicity. In addition, glucotoxicity can cause oxidative stress, inflammation, fibrosis, apoptosis, and cell injury in a variety of tissues. As a result, glucotoxicity plays a significant indirect role in hyperglycaemia-induced histological damage in a variety of organs, including the respiratory system. ${ }^{24}$

COVID-19 is hypothesized to induce tissue damage in a hyperglycemic environment and promote other pathophysiological processes. Codo et al. recently found that raised glucose levels promoted COVID-19 infection via a hypoxia-induced factor-1- (HIF-1-) depending mechanism. They claimed that due of the harmful consequences of hyperglycemia, individuals with diabetes were more vulnerable to SARS-CoV2 infection. 25 The increased severity of symptoms and tissue injury in the respiratory system seen in individuals with diabetes who were caught with SARS-CoV2 are strongly connected to the harmful result of hyperglycemia, which trigger numerous toxic pathways and harm infected host cells.
Therefore, maintaining a patient's glucose homeostasis might greatly lower the severity of COVID-19 infection and the incidence of disability and mortality by avoiding cellular damage promoted by glucotoxicity. ${ }^{15}$

\section{Stress on the Endoplasmic Reticulum (ER)}

Endoplasmic reticulum (ER) is a cellular organelle that synthesizes and transports macromolecules such as lipids, proteins, and carbohydrates. It is also contributed in the producing and maturation of these biomolecules by folding, glycosylation, and the production of disulfide bonds. In order to maintain cellular homeostasis, this essential organelle delivers biomolecules to their proper destination. Any decrease in ER activity is associated with the accretion of misfolded proteins in the ER lumen, inducing in a pathologic condition known as "ER stress". ${ }^{15}$

Endoplasmic reticulum stress is a typical occurrence in diabetics. Besides that, ER stress has a substantial relationship with coronavirus activity. According to several studies, the coronavirus causes ER stress by upregulating the $\mathrm{S}$ (spike) protein and enabling virus entrance into cells which promotes replication and enhances the coronavirus's infectivity and pathogenicity. ${ }^{15,26}$

Although there is little evidence, researchers thought that SARS-CoV2 pathogenicity is higher in diabetes individuals, which may be due to pre-existing ER stress in diabetic patients, which may increase viral entry and high viral pathogenicity. ${ }^{15}$

\section{Changes in the Renin-Angiotensin-Aldosterone System (RAAS)}

The renin-angiotensin-aldosterone system (RAAS) regulates fluid and electrolytes as well as systemic vascular resistance in our body." The mechanism is activated by the release of renin from the kidneys," "which is followed by renin cleaving angiotensinogen into angiotensin I (Ang I)." The angiotensin-converting enzyme (ACE) converts Ang I to angiotensin II (Ang II) "on the surface of vascular endothelial cells in the lungs and proximal renal tubules." While ACE catalyzes this conversion, ACE2 counteracts it by boosting Ang 1-7 and reducing Ang II activity. This system's major end effector, Ang II, is a strong vasoconstrictive peptide. It primarily operates by attaching to two types of receptors known as type 1 (AT1) and type 2 (AT2) (AT2). Furthermore, it stimulates the secretion of aldosterone, a hormone included in electrolyte balance. ${ }^{27}$

SARS-CoV2 interacts closely with RAAS activity. The virus penetrates cells via interacting with the spike (S) protein on ACE2 receptors. People with diabetes have some level of RAAS dysfunction, and ACE inhibitors or ARBs (Angiotensin receptor blockers) are commonly used to "avoid along with manage diabetes-related vascular problems." In hypertensive individuals with impaired RAAS function, these medications consistently demonstrated protective benefits for pulmonary sequelae and the risk of lung infection. As a result, it may be predicted that individuals with diabetes are more likely to be vulnerable to COVID-19 with severe symptoms due to the RAAS function, which can enhance viral entrance into cells. However, some contradictory evidence suggests that ACE inhibitors or ARBs "have no relationship with the likelihood" of COVID-19 infection. Pharmacological treatments targeting RAAS have the potential to decrease the bad effect of SARS-CoV2 in diabetic individuals. 


\section{Apoptosis}

Also known as programmed cell destruction, Apoptosis is a cellular event that happens physiologically throughout numerous mechanism such as development, maturation, and migration. Apoptosis, along with necrosis and fibrosis, "is responsible for" the majority of tissue damage and dysfunction caused by cellular termination. Diabetes Mellitus is typically linked with a greater rate of cell death related to apoptosis, according to numerous studies. Proapoptotic factors are increased in patients with uncontrolled hyperglycemia. ${ }^{15}$

Apoptosis is also crucial in the tissue damage created by the coronavirus. "It is directly engaged in cell death and tissue damage caused by" coronavirus infections. As a result, apoptosis may be one of the most possible explanations for patients with diabetes who were caught with SARS-CoV2 had more serious tissue damage and cell deaths than those without diabetes. ${ }^{26}$

\section{Other mechanism}

Aside from the processes outlined above, mitochondrial dysfunction in diabetic individuals is another potential route with minimal clinical evidence. Mitochondrial dysfunction is prevalent in uncontrolled diabetes. Mitochondrial damage occurs in viral infections as well, such as coronavirus. This might be one of the probable link between diabetes and COVID-19 severity. ${ }^{28,29}$

\section{Diabetes Mellitus with New Onset in COVID-19}

Several clinical researches reveal that COVID-19 can induce new-onset diabetes. This can happen even in people who do not have any underlying conditions for poor glucose metabolism. The underlying processes have been identified as impaired pancreatic insulin secretion and insulin resistance. In COVID-19 patients, new-onset hyperglycemia is correlated with a poor outcome. In fact, the prognosis may be worse than in people who already have diabetes. ${ }^{30}$

Diabetes Mellitus has been discovered as an independent factor influencing COVID-19 patients' poor prognosis. Diabetic was shown to be prevalent in patients with no history of DM or prior glucocorticoid usage during the SARS-CoV-1 outbreak, and it was an independent predictor of patient death. Diabetes Mellitus has been correlated to COVID-19, which is caused by the SARS-CoV-2 virus. New-onset hyperglycemia and acute metabolic decompensation from pre-existing diabetes are showing significant COVID-19 problems, particularly in hospitalized patients. Other risk factors, including obesity, pre-diabetes problems, a history of DM, or corticosteroid medication, are not linked with new-onset hyperglycemia. These evidence points to a two-way connection among diabetes and COVID-19. ${ }^{31}$

Ceriello et al and Apicella et al investigated the pathogenic pathways that might lead to new-onset hyperglycemia in COVID-19 patients. The most likely underlying mechanisms are insulin resistance and insulin secretion abnormalities, both of which can play a role in causing new-onset hyperglycemia in COVID-19 individuals, even if they do not have a history of diabetes. $^{32,33}$ SARS-CoV-2 can penetrate pancreatic (beta) islet cells via the production of angiotensin-converting enzyme 2 (ACE2) receptors. High levels of inflammatory cytokines (such as IL-6 and TNF-) caused by SARS-CoV-2 infection can cause structural and functional abnormalities in endothelial cells, leading to insulin secretion dysfunction and pancreatic beta cell damage, resulting in an increase in blood glucose, which aggravates or even triggers newonset of Diabetes Mellitus..$^{17,34,35}$

Huiqing $\mathrm{Li}$ et al discovered that the death rate in the COVID-19 patients with new-onset Diabetes Mellitus was greater than in the COVID-19 patients with previously known to have Diabetes Mellitus. "Based on these findings, a worldwide list of COVID-19 patients with new-onset DM has been developed by many diabetes specialists to further study the complexities and consequences of this connection. ${ }^{36}$

\section{Conclusion}

$\mathrm{DM}$ is now recognized to be a comorbid linked with a poorer outcome of COVID-19. Recent investigations have shown that COVID-19 can cause hyperglycemia or new-onset Diabetes Mellitus with a poorer prognosis than COVID-19 patients with pre-existing diabetes. Further study is needed to understand, prevent, and treat this new disease.

\section{Conflict of Interest}

There is no conflict of interest to declare.

\section{References}

1. Zhou P, Yang X-L, Wang X-G, Hu B, Zhang L, Zhang W. A pneumonia outbreak associated with a new coronavirus of probable bat origin. Nature. 2020;579(7798):270-3.

2. Azer SA. COVID-19: pathophysiology, diagnosis, complications and investigational therapeutics. New Microbes New Infect [Internet]. 2020;37(M):100738. Available from: https://doi.org/10.1016/j. nmni.2020.100738

3. WHO. COVID-19 Weekly Epidemiological Update. World Heal Organ [Internet]. 2020;(November):1;4. Available from: https://www.who.int/docs/default-source/coronaviruse/situationreports/20201012-weekly-epi-update-9.pdf

4. WHO. Coronavirus Disease 2019 (COVID-19) World Health Situation Report - 1. WHO Indones Situat Rep. 2020;2019(March):8.

5. Lotfi M, Hamblin MR, Rezaei N. COVID-19: Transmission, prevention, and potential therapeutic opportunities. Clin Chim Acta. 2020 Sep;508:254-66.

6. Yuki K, Fujiogi M, Koutsogiannaki S. COVID-19 pathophysiology: A review. Clin Immunol. 2020;215(April).

7. Wiersinga WJ, Rhodes A, Cheng AC, Peacock SJ, Prescott HC. Pathophysiology, Transmission, Diagnosis, and Treatment of Coronavirus Disease 2019 (COVID-19). JAMA. 2020 Aug $25 ; 324(8): 782$.

8. Gandhi RT, Lynch JB, del Rio C. Mild or Moderate Covid-19. Solomon CG, editor. N Engl J Med. 2020 Oct 29;383(18):1757-66.

9. Rothe C, Schunk M, Sothmann P, Bretzel G, Froeschl G, Wallrauch C, et al. Transmission of 2019-nCoV Infection from an Asymptomatic Contact in Germany. N Engl J Med. 2020;382(10):970-1.

10. Li Q, Guan X, Wu P, Wang X, Zhou L, Tong Y, et al. Early Transmission Dynamics in Wuhan, China, of Novel CoronavirusInfected Pneumonia. N Engl J Med. 2020;382(13):1199-207.

11. Zhou F, Yu T, Du R, Fan G, Liu Y, Liu Z, et al. Clinical course and risk factors for mortality of adult inpatients with COVID-19 in Wuhan, China: a retrospective cohort study. Lancet. 2020 Mar;395(10229):1054-62.

12. Sousa GJB, Garces TS, Cestari VRF, Florêncio RS, Moreira TMM, Pereira MLD. Mortality and survival of COVID-19. Epidemiol Infect. 2020;

13. Palaiodimos L, Chamorro-Pareja N, Karamanis D, Li W, Zavras PD, Chang KM, et al. Diabetes is associated with increased risk for inhospital mortality in patients with COVID-19: a systematic review and meta-analysis comprising 18,506 patients. Hormones. 2020 Oct 29 ;

14. Guo W, Li M, Dong Y, Zhou H, Zhang Z, Tian C, et al. Diabetes is a risk factor for the progression and prognosis of $<$ scp $>$ COVID $</$ scp $>$ -19. Diabetes Metab Res Rev. 2020 Oct 7;36(7):1-9. 
15. Yaribeygi H, Sathyapalan T, Jamialahmadi T, Sahebkar A. The Impact of Diabetes Mellitus in COVID-19: A Mechanistic Review of Molecular Interactions. J Diabetes Res. 2020;2020.

16. Shang L, Shao M, Guo Q, Shi J, Zhao Y, Xiaokereti J, et al. Diabetes Mellitus is Associated with Severe Infection and Mortality in Patients with COVID-19: A Systematic Review and Meta-analysis. Arch Med Res. 2020;51(7):700-9.

17. Chen L, Deng H, Cui H, Fang J, Zuo Z, Deng J, et al. Inflammatory responses and inflammation-associated diseases in organs. Oncotarget. 2018;9(6):7204-18.

18. Elliott MR, Koster KM, Murphy PS. Efferocytosis Signaling in the Regulation of Macrophage Inflammatory Responses. J Immunol. 2017;198(4):1387-94.

19. Yaribeygi H, Atkin SL, Pirro M, Sahebkar A. A review of the antiinflammatory properties of antidiabetic agents providing protective effects against vascular complications in diabetes. J Cell Physiol. 2019;234(6):8286-94.

20. Prompetchara E, Ketloy C, Palaga T. Immune responses in COVID-19 and potential vaccines: Lessons learned from SARS and MERS epidemic. Asian Pacific J Allergy Immunol. 2020;38(1):1-9.

21. Ye Q, Wang B, Mao J. The pathogenesis and treatment of the 'Cytokine Storm' in COVID-19. J Infect. 2020 Jun;80(6):607-13.

22. Yaribeygi H, Sathyapalan T, Atkin SL, Sahebkar A. Molecular Mechanisms Linking Oxidative Stress and Diabetes Mellitus. Oxid Med Cell Longev. 2020 Mar 9;2020:1-13.

23. Lim Y, Ng Y, Tam J, Liu D. Human Coronaviruses: A Review of Virus-Host Interactions. Diseases. 2016 Jul 25;4(4):26.

24. Luo X, Wu J, Jing S, Yan L. Hyperglycemic Stress and Carbon Stress in Diabetic Glucotoxicity. 2016;7(1):1-21.

25. Codo A, Davanzo G, Monteiro L. Elevated Glucose Levels Favor SARS-CoV-2 Infection and Monocyte Response through a HIF-1 $\alpha /$ Glycolysis-Dependent Axis. Cell Metab. 2020;32(3):437-46.

26. Fung TS, Liu DX. Coronavirus infection, ER stress, apoptosis and innate immunity. Front Microbiol. 2014 Jun 17;5(296).
27. Santos RAS, Sampaio WO, Alzamora AC, Motta-Santos D, Alenina $\mathrm{N}$, Bader M, et al. The ACE2/Angiotensin-(1-7)/MAS Axis of the Renin-Angiotensin System: Focus on Angiotensin-(1-7). Physiol Rev. 2018 Jan 1;98(1):505-53.

28. Khan M, Syed GH, Kim S, Siddiqui A. Mitochondrial dynamics and viral infections: A close nexus. Biochim Biophys Acta - Mol Cell Res. 2015 Oct;1853(10):2822-33.

29. Luo W-R, Yu H, Gou J-Z, Li X-X, Sun Y, Li J-X, et al. Histopathologic Findings in the Explant Lungs of a Patient With COVID-19 Treated With Bilateral Orthotopic Lung Transplant. Transplantation. 2020 Nov 24;104(11):e329-31.

30. Papachristou S, Stamatiou I, Stoian AP, Papanas N. New-Onset Diabetes in COVID-19: Time to Frame Its Fearful Symmetry. Diabetes Ther. 2021 Feb 26;12(2):461-4.

31. Singh AK, Singh R. Hyperglycemia without diabetes and new-onset diabetes are both associated with poorer outcomes in COVID-19. Diabetes Res Clin Pract. 2020 Sep;167(August):108382.

32. Ceriello A, De Nigris V, Prattichizzo F. Why is hyperglycaemia worsening COVID-19 and its prognosis? Diabetes, Obes Metab. 2020;22(10):1951-2.

33. Apicella M, Campopiano MC, Mantuano M, Mazoni L, Coppelli A, Del Prato S. COVID-19 in people with diabetes: understanding the reasons for worse outcomes. Lancet Diabetes Endocrinol. 2020 Sep;8(9):782-92.

34. Zhou W, Ye S, Wang W, Li S, Hu Q. Clinical Features of COVID-19 Patients with Diabetes and Secondary Hyperglycemia. J Diabetes Res. 2020;2020(Dm).

35. Maddaloni E, Buzzetti R. Covid-19 and diabetes mellitus: unveiling the interaction of two pandemics. Diabetes Metab Res Rev. 2020;36(7):19-20.

36. Li H, Tian S, Chen T, Cui Z, Shi N, Zhong X, et al. Newly diagnosed diabetes is associated with a higher risk of mortality than known diabetes in hospitalized patients with COVID-19. Diabetes, Obes Metab. 2020;22(10):1897-906. 